\title{
Almost Properness of Extremal Mappings
}

\author{
by \\ Armen EDIGARIAN and Przemysław KLIŚ
}

Presented by Józef SICIAK

Summary. We give a simple proof of almost properness of any extremal mapping in the sense of Lempert function or in the sense of Kobayashi-Royden pseudometric.

Let $D \subset \mathbb{C}^{n}$ be a domain. For any $z, w \in D$ (resp. $z \in D, X \in \mathbb{C}^{n}$ ) we denote by $\sigma_{1}(z, w)\left(\right.$ resp. $\left.\sigma_{2}(z, X)\right)$ the set of all points $r \in[0,1)$ (resp. $r \geq 0$ ) such that we can find a holomorphic mapping from the unit disc $\mathbb{D}$ to $D$ with $f(0)=z$ and $f(r)=w\left(\right.$ resp. $\left.r f^{\prime}(0)=X\right)$. We put

$$
\tilde{k}_{D}(z, w)=\inf _{r \in \sigma_{1}(z, w)} r \text { and } \kappa_{D}(z, X)=\inf _{r \in \sigma_{2}(z, X)} r .
$$

We call $\widetilde{k}_{D}$ the Lempert function and $\kappa_{D}$ the Kobayashi-Royden pseudometric (see e.g. [3]). A holomorphic mapping $f: \mathbb{D} \rightarrow D$ is a $\widetilde{k}_{D^{-}}$extremal (resp.

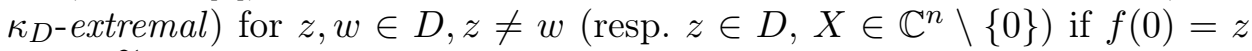
and $f\left(\widetilde{k}_{D}(z, w)\right)=w\left(\right.$ resp. $f(0)=z$ and $\left.\kappa_{D}(z, X) f^{\prime}(0)=X\right)$.

The Lempert function and the Kobayashi-Royden pseudometric play an essential role in complex analysis, especially in problems related to boundary properties of biholomorphic (more generally, proper holomorphic) mappings (see e.g. [2]). In many cases, the primary problem is to show that appropriate bounded extremal functions $f: \mathbb{D} \rightarrow D$ are almost proper, i.e., $f^{*}(\zeta) \subset \partial D$ for a.a. $\zeta \in \mathbb{T}$, where $\mathbb{T}$ denotes the unit circle and $f^{*}$ denotes the nontangential boundary value of $f$ (see e.g. [6]). This problem was studied for example in [4], [5], [1], [3]. The main idea of the paper is to give a truly elementary proof of a result from [5] in a more general setting.

2000 Mathematics Subject Classification: Primary 32F45.

Key words and phrases: Kobayashi-Royden metric, Lempert function, extremal mapping, almost proper mapping. 
Theorem 1. Let $D \Subset \mathbb{C}^{n}$ be a weakly Runge domain (see below) and let $f: \mathbb{D} \rightarrow D$ be a holomorphic mapping such that for some $\gamma>0$ we have

$$
\operatorname{dist}(f(\lambda), \partial D) \geq \gamma(1-|\lambda|), \quad \lambda \in \mathbb{D} .
$$

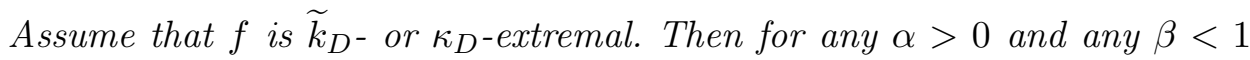
the set

$$
\left\{\lambda \in \mathbb{T}: \operatorname{dist}(f(t \lambda), \partial D) \geq \alpha(1-t)^{\beta}, t \in(0,1)\right\}
$$

has Lebesgue measure zero in $\mathbb{T}$. In particular, $f^{*}(\zeta) \in \partial D$ for a.a. $\zeta \in \mathbb{T}$.

We say that $D \Subset \mathbb{C}^{n}$ is a weakly Runge domain if there exists a domain $G \supset \bar{D}$ such that for any bounded holomorphic mapping $f: \mathbb{D} \rightarrow G$ with $f^{*}(\mathbb{T}) \Subset D$ we have $f(\mathbb{D}) \Subset D$.

Proof of Theorem 1. For $\alpha>0$ and $\beta<1$ we put

$$
Q(\alpha, \beta)=\left\{\lambda \in \mathbb{T}: \operatorname{dist}(f(t \lambda), \partial D) \geq \alpha(1-t)^{\beta}, t \in(0,1)\right\} .
$$

Note that for any $\beta_{1}<\beta_{2}$ we have $Q\left(\alpha, \beta_{1}\right) \subset Q\left(\alpha, \beta_{2}\right)$.

So, without loss of generality we may assume that for some $\alpha>0$ and $\beta \in(0,1)$ the set $Q(\alpha, \beta)$ has positive measure. In the following we denote the set $Q(\alpha, \beta)$ by $P$. We may assume that $0<(2 \pi)^{-1} \int_{P} d \theta<1$ (otherwise we take as $P$ any smaller subset of $Q(\alpha, \beta)$ of positive measure). We put

$$
\varphi(z)=\frac{1}{2 \pi} \int_{P} \frac{e^{i \theta}+z}{e^{i \theta}-z} d \theta .
$$

Note that $0<\Re \varphi(\lambda)<1$ for any $\lambda \in \mathbb{D}$. Without loss of generality, we may assume that $f$ is $\widetilde{k}_{D^{-}}$extremal for points $f(0), f(\sigma)$ (resp. $\kappa_{D}$-extremal for $\left.f(0), f^{\prime}(0)\right)$. For a fixed $t \in(0,1)$ consider a mapping

$$
g_{t}(\lambda)=f(t \lambda)+e^{\gamma_{t}(\varphi(\lambda)-\varphi(\sigma))} \frac{\lambda}{\sigma}(f(\sigma)-f(t \sigma))
$$

(resp. $\left.g_{t}(\lambda)=f(t \lambda)+e^{\gamma_{t}(\varphi(\lambda)-\varphi(0))} \lambda(1-t) f^{\prime}(0)\right)$, where $\gamma_{t} \in \mathbb{R}$ will be chosen later. Note that $g_{t}(0)=f(0)$ and $g_{t}(\sigma)=f(\sigma)$ (resp. $g_{t}(0)=f(0)$ and $\left.g_{t}^{\prime}(0)=f^{\prime}(0)\right)$. Our aim is to show that for all $t \in(0,1)$ sufficiently close to 1 we can choose $\gamma_{t}$ in such a way that $g_{t}(\mathbb{D}) \Subset D$, which contradicts the extremality of $f$. To get this we only have to show that $g_{t}^{*}(\mathbb{T}) \Subset D$. We will prove this for $\widetilde{k}_{D^{-}}$extremal mappings (for $\kappa_{D^{-}}$extremals one can use similar arguments).

It is sufficient to show that for any $t$ close to 1 we have

$$
\left\|e^{\gamma_{t}\left(\varphi^{*}(\lambda)-\varphi(\sigma)\right)} \frac{\lambda}{\sigma}(f(\sigma)-f(t \sigma))\right\| \leq \frac{\alpha}{2}(1-t)^{\beta} \quad \text { for } \lambda \in P
$$

and

$$
\left\|e^{\gamma_{t}\left(\varphi^{*}(\lambda)-\varphi(\sigma)\right)} \frac{\lambda}{\sigma}(f(\sigma)-f(t \sigma))\right\| \leq \frac{\gamma}{2}(1-t) \quad \text { for } \lambda \in \mathbb{T} \backslash P .
$$


Since $\|f(\sigma)-f(t \sigma)\| \leq \rho|\sigma|(1-t)$, it suffices to have

$$
e^{\gamma_{t}(1-\Re \varphi(\sigma))} \rho \leq \frac{\alpha}{2}(1-t)^{\beta-1}
$$

and

$$
e^{-\gamma_{t} \Re \varphi(\sigma)} \rho \leq \frac{\gamma}{2}
$$

Take $\gamma_{t}$ such that

$$
e^{\gamma_{t}(1-\Re \varphi(\sigma))} \rho=\frac{\alpha}{2}(1-t)^{\beta-1} .
$$

Then for $t$ sufficiently close to 1 we also have inequality (4). Moreover,

$$
\left\|g_{t}-f(t \cdot)\right\|_{\mathbb{D}} \rightarrow 0 \quad \text { as } t \rightarrow 1 .
$$

Since $D$ is a weakly Runge domain, $g_{t}(\mathbb{D}) \Subset D$ for $t$ close enough to 1 .

To end the proof suppose that there exists a set $P \subset \mathbb{T}$ of positive measure such that for all $\zeta \in P$ we have

$$
\operatorname{dist}\left(f^{*}(\zeta), \partial D\right)>\epsilon>0 .
$$

Put

$$
P_{n}=\{\lambda \in \mathbb{T}: \operatorname{dist}(f(t \lambda), \partial D)>\epsilon \text { for any } t \in(1-1 / n, 1)\}, \quad n \in \mathbb{N} .
$$

Note that $P \subset \bigcup_{n \in \mathbb{N}} P_{n}$. Hence, for some $n_{0}$ the set $P_{n_{0}}$ is of positive measure.

REMARK 2. (i) Note that any Runge domain is weakly Runge.

(ii) Take any domain $G \subset \mathbb{C}^{n}$ and let $u$ be a plurisubharmonic function in $G$. Assume that $D=\{z \in G: u(z)<0\} \Subset G$. Then $D$ is weakly Runge.

Let us show that (2) holds for any analytic disc in a large class of domains.

Definition 3 (see [5]). A domain $D \subset \mathbb{C}^{n}$ is called $\rho$-pseudoconvex if there is a $\rho \in \mathrm{PSH} \cap \mathcal{C}(\bar{D})$ such that $\left.\rho\right|_{\partial D}=0, \rho<0$ on $D$ and $\operatorname{dist}(z, \partial D)$ $\geq|\rho(z)|$.

Proposition 4. Let $D \subset \mathbb{C}^{n}$ be a $\rho$-pseudoconvex domain and let $f$ : $\mathbb{D} \rightarrow D$ be an analytic disc. Then (2) is satisfied.

Proof. Let $\rho$ be a plurisubharmonic function given by the definition of the $\rho$-psedoconvex domain. Consider the subharmonic function $v=\rho \circ f$. Note that for some $C>0$ we have $|\rho(f(\zeta))| \geq C(1-|\zeta|)$ (see e.g. [5]), and therefore $\operatorname{dist}(f(\zeta), \partial D) \geq C(1-|\zeta|)$.

Note that if $D_{1}, D_{2}$ are bounded domains and $D$ is any connected component of $D_{1} \cap D_{2}$, and if $f: \mathbb{D} \rightarrow D$ is such that $\operatorname{dist}\left(f(\lambda), \partial D_{j}\right) \geq \gamma_{j}(1-|\lambda|)$ for $j=1,2$ and $\lambda \in \mathbb{D}$ then $\operatorname{dist}(f(\lambda), \partial D) \geq \min \left\{\gamma_{1}, \gamma_{2}\right\}(1-|\lambda|)$. The class of $\rho$-pseudoconvex domains contains in particular the strongly pseudoconvex domains and the analytic polyhedra, i.e., bounded connected components of 
sets $\left\{z \in \mathbb{C}^{n}:\left|f_{j}(z)\right|<1, j=1, \ldots, m\right\}$, where $f_{j}, j=1, \ldots, m$, are holomorphic functions in $\mathbb{C}^{n}$.

REMARK 5. In the proof of Theorem 2 in [5], E. Poletsky used the fact that if $D \Subset \mathbb{C}^{n}$ is a $\rho$-pseudoconvex domain and if $f: \mathbb{D} \rightarrow \mathbb{C}^{n}$ is a bounded holomorphic mapping such that $f^{*}(\zeta) \in D$ for a.a. $\zeta \in \mathbb{T}$, then $f(\mathbb{D}) \subset D$. Note that this is not true for annuli on the complex plane (which are $\rho$-pseudoconvex and weakly Runge). That is why in Theorem 1 we assume more, namely a Runge type property.

REMARK 6. W. Zwonek [7] constructed a pseudoconvex Reinhardt domain $D$ and an extremal mapping $f: \mathbb{D} \rightarrow D$ for which (2) is not satisfied. Consider the domain $D=\left\{(z, w) \in \mathbb{D}^{2}:|w|<e^{|z| /(|z|-1)}\right\}$ and the holomorphic mapping $f(\lambda)=(\lambda, 0)$. Then $D$ is a pseudoconvex Reinhardt domain and $f: \mathbb{D} \rightarrow D$ is an extremal mapping. However, (2) is not satisfied.

Corollary 7. Let $G \subset \mathbb{C}^{n}$ be a domain and let $f_{1}, \ldots, f_{m}$ be holomorphic functions such that

$$
\widetilde{G}=\left\{z \in G:\left|f_{j}(z)\right|<1, j=1, \ldots, m\right\} \Subset G .
$$

If $D$ is any connected component of $\widetilde{G}$ then any $\widetilde{k}_{D^{-}}$and $\kappa_{D^{-e x t r e m a l}}$ is almost proper.

Remark 8. Note that if $D \subset \mathbb{C}$ is a taut domain, i.e., different from $\mathbb{C}$ and $\mathbb{C} \backslash\{a\}, a \in \mathbb{C}$, then any $k_{D^{-}}$and $\kappa_{D^{-}}$extremal $f: \mathbb{D} \rightarrow D$ is a covering (see e.g. [3]). Therefore, $f$ is almost proper. We do not know whether for any taut domain in $\mathbb{C}^{n}$ its extremal mappings are almost proper.

Using the above technique we can show the following property of $\widetilde{k}_{D^{-}}$ extremals.

Proposition 9. Let $D \Subset \mathbb{C}^{n}$ be a domain and let $f: \mathbb{D} \rightarrow D$ be a holomorphic mapping such that for some $\gamma>0$ we have

$$
\operatorname{dist}(f(\lambda), \partial D) \geq \gamma(1-|\lambda|), \quad \lambda \in \mathbb{D} .
$$

Assume that $f$ is $\widetilde{k}_{D}$-extremal for $(f(0), f(\sigma))$. Then $f^{\prime}(\sigma) \neq 0$.

Proof. For a fixed $t \in(0,1)$ consider a mapping

$$
g_{t}(\lambda)=f(t \lambda)+\frac{\lambda}{\sigma}(f(\sigma)-f(t \sigma)) .
$$

Note that $g_{t}(0)=f(0)$ and $g_{t}(\sigma)=f(\sigma)$. Assume that $f^{\prime}(\sigma)=0$. We want to show that for $t$ sufficiently close to 1 we have $g_{t}(\mathbb{D}) \Subset D$. Indeed, put

$$
\psi_{t}(\lambda)=\lambda \frac{f(\sigma)-f(t \sigma)}{\sigma(1-t)} .
$$

Then $\left\|\psi_{t}\right\|_{\mathbb{D}} \rightarrow 0$ as $t \rightarrow 1$. Hence, for $t$ sufficiently close to 1 we have $\left\|\psi_{t}\right\|_{\mathbb{D}} \leq \gamma / 2$. 
Acknowledgements. The authors thank Professor Włodzimierz Zwonek for helpful remarks.

The first author was supported in part by the Polish Ministry of Science and Higher Education Grant No. N N201 361436.

\section{References}

[1] A. Edigarian, On extremal mappings in complex ellipsoids, Ann. Polon. Math. 62 (1995), 83-96.

[2] F. Forstnerič and J.-P. Rosay, Localizations of the Kobayashi metric and the boundary continuity of proper holomorphic mappings, Math. Ann. 279 (1987), 239-252.

[3] M. Jarnicki and P. Pflug, Invariant Distances and Metrics in Complex Analysis, de Gruyter, 1993.

[4] L. Lempert, La métrique de Kobayashi et la représentation des domaines sur la boule, Bull. Soc. Math. France 109 (1981), 427-474.

[5] E. Poletsky, The Euler-Lagrange equations for extremal holomorphic mappings of the unit disk, Michigan Math. J. 30 (1983), 317-333.

[6] W. Rudin, Real and Complex Analysis, 2nd ed., McGraw-Hill, New York, 1974.

[7] W. Zwonek, personal communication.

Armen Edigarian and Przemysław Kliś

Institute of Mathematics

Jagiellonian University

Łojasiewicza 6

30-348 Kraków, Poland

E-mail: armen.edigarian@im.uj.edu.pl

przemyslaw.klis@im.uj.edu.pl

Received May 25, 2009;

received in final form June 10, 2009 